\title{
Development of a Process Analytical Technology (PAT) for in-line monitoring of film thickness and mass of coating materials during a pan coating operation
}

\author{
Claire Gendre ${ }^{\mathrm{a}, \mathrm{b}, \mathrm{c}, *}$, Muriel Genty ${ }^{\mathrm{a}}$, Mathieu Boiret ${ }^{\mathrm{a}}$, Marc Julien ${ }^{\mathrm{a}}$, Loïc Meunier ${ }^{\mathrm{a}}$, Olivier Lecoq ${ }^{\mathrm{b}}$, \\ Michel Baron ${ }^{b}$, Pierre Chaminade ${ }^{c}$, Jean Manuel Péan ${ }^{a}$ \\ ${ }^{a}$ Technologie SERVIER, 27 rue Eugène Vignat, 45000 Orléans, France \\ ${ }^{\mathrm{b}}$ Université de Toulouse, Mines Albi, RAPSODEE FRE CNRS 3213, 81013 Albi, France \\ ' Univ Paris-Sud, EA 4041, Groupe de Chimie Analytique de Paris-Sud, IFR 141-IPSIT, Faculté de Pharmacie, 92296 Châtenay-Malabry, France
}

Keywords:

Process Analytical Technology (PAT)

Near Infrared Spectroscopy (NIRS)

In-line measurements

Real-time monitoring

Coating

Terahertz Pulsed Imaging (TPI)

\begin{abstract}
A B S T R A C T
The aim of this study was to perform in-line Near Infrared (NIR) measurements inside a pan coater to monitor a coating operation in real-time, by predicting the increases in mass of coating materials and coating thickness. A polymer combination of ethylcellulose/poly(vinyl-alcohol)-poly(ethylene-glycol) graft copolymer was used as functional aqueous coating. Coated tablets were sampled at regular intervals during the coating operation, then subjected to either simple and fast weighing $(n=50)$ or accurate and non-destructive Terahertz Pulsed Imaging (TPI) measurements $(n=3)$. Off-line NIR spectra analysis revealed that the coating operation could efficiently be controlled by focusing on two distinct NIR regions, related to absorption bands of ethylcellulose. Principal component analysis of in-line NIR spectra gave a clear classification of the collected coated tablets. Real-time quantitative monitoring of the coating operation was successfully performed from partial least square calibration models built using either TPI or weighing as reference method. Coating thicknesses as well as mass of coating materials used as primary values provided accurate NIR predictions. A comparison study demonstrated that both reference methods led to reliable and accurate real-time monitoring of the coating operation. This work demonstrated that in-line NIR measurements associated with multivariate analyses can be implemented to monitor in real-time a pan coating operation in order to fulfil the expectations of ICH Q8 guideline on pharmaceutical development, especially in terms of PAT control strategy and reduced end-product testing.
\end{abstract}

\section{Introduction}

Aqueous-based coating is one of the strategies frequently used for controlled drug release applications (McGinity and Felton, 2008). The polymer coating is applied to tablets to decrease drug release rate and to obtain a continuous release profile. Homogeneity and thickness of the coating are well-known to be critical regarding drug release rate (Porter et al., 2009). According to ICH Q8 guideline on pharmaceutical development (ICH Q8 R2, 2009), introducing the Quality by Design approach, these critical quality attributes should be controlled and monitored to ensure that the product is of the desired quality. Process analytical technologies allow timely measurements of critical quality and performance attributes of raw and in-process materials (Food and Drug Administration, 2004). Especially, Near Infrared (NIR)

\footnotetext{
* Corresponding author at: Technologie SERVIER, 27 rue Eugène Vignat, 45000 Orléans, France. Tel.: +33 (0)2 382380 00; fax: +33 (0)2 38238201.

E-mail address: claire.gendre@gmail.com (C. Gendre).
}

spectroscopy, a versatile vibrational spectroscopy technique for pharmaceutical analysis (Reich, 2005), was previously proposed for real-time control and monitoring of thickness and coating uniformity over the whole coating operation. So far, only a few articles described real-time measurements performed in-line by NIR spectroscopy during coating operations. Andersson et al. (2000) and Lee et al. $(2010,2011)$ reported in-line NIR measurements for fluid bed coating of pellets, while Perez-Ramos et al. (2005) and Römer et al. (2008) performed in-line analyses for pan coating of tablets. The difficulties to integrate a measuring system inside a coater apparatus and the required fast sampling acquisition were pointed out (Perez-Ramos et al., 2005).

To extract relevant information from the NIR multivariate signal, appropriate chemometric tools are requested, as well as primary measurements to calibrate NIR spectral information (Roggo et al., 2007). Different reference methods were studied to predict the amount of polymer applied to tablets. Weighing was used as reference method by Han and Faulker (1996) and Kirsch and Drennen (1996). This fast and simple approach requires no sample 
preparation. Nevertheless, a major drawback in terms of accuracy remains its dependency on core tablet weight uniformity. For this reason, tablet coating thickness values were predominantly used. Various techniques for coating thickness determination were employed. However, these techniques also present some drawbacks. For instance, optical microscopy and image analysis are destructive and require time-consuming sample preparation (Andersson et al., 1999, 2000; Cahyadi et al., 2010; Larsen et al., 2003). Several studies reported tablet thickness measurements using a micrometer (Kirsch and Drennen, 1995; Lee et al., 2010; Moes et al., 2008; Perez-Ramos et al., 2005; Römer et al., 2008; Tabasi et al., 2008). Although this coating thickness determination technique is fast, it is less accurate as the dimension variability of core tablets is taken into account. Terahertz Pulsed Imaging (TPI) was described as a powerful alternative for coating thickness determination due to its non-destructive nature and its ability to provide a three dimension map of coating thickness over the whole tablet surface (Fitzgerald et al., 2005; Ho et al., 2007; Zeitler et al., 2007). Some authors concluded that this method could only be applied to the monitoring of thickness values higher than $35 \mu \mathrm{m}$ due to the detection limit of the instrument (Cogdill et al., 2007; Maurer and Leuenberger, 2009). For the present study, since specifications for dissolution rate requires high polymer deposits (Siepmann et al., 2007), TPI could be selected as a relevant reference method.

A functional polymer combination of ethylcellulose/poly(vinylalcohol)-poly(ethylene-glycol) graft copolymer, using triethylcitrate as plasticizer, was previously studied for aqueous coating of pellets (Muschert et al., 2009). In the present work, this polymer blend was applied to tablets during a pan coating operation. To our knowledge, up until now in-line NIR measurements for real-time monitoring of a tablet coating operation with this coating suspension have never been reported.

The objectives of this work were thus to demonstrate the feasibility of real-time tablet coating monitoring by implementation of a near infrared probe inside a pan coater and to compare two distinct reference methods. Off-line NIR spectra analysis was first performed to highlight absorption bands characteristic of core tablet or coating materials. Then, qualitative in-line analysis aimed at demonstrating the ability of NIRS to monitor the coating operation. Quantitative NIR models were developed using either weighing or TPI as reference method to calibrate NIR spectral information. Finally, NIR predictions obtained from NIR calibration models based on both primary measurements were compared.

\section{Material and methods}

\subsection{Core tablets and coating materials}

Core tablets of biconvex shape (height of $4 \mathrm{~mm}$, diameter of $8 \mathrm{~mm}$, curvature radius of $7 \mathrm{~mm}$ and an average mass of $200 \mathrm{mg}$ ), containing a freely soluble drug substance, were supplied by Les Laboratoires Servier (Gidy, France).

Aqueous ethylcellulose dispersion (Aquacoat ECD 30 ${ }^{\circledR}$, FMC Biopolymer, Philadelphia, USA), poly(vinyl-alcohol)-poly(ethylene-glycol) graft copolymer (PVA-PEG, Kollicoat $\mathrm{IR}^{\circledR}$, BASF, Ludwigshafen, Germany) and triethylcitrate (TEC, Citroflex $2^{\circledR}$, Morflex, Greensboro, USA) were purchased as coating materials.

Ethylcellulose powder (EC, Ethocel ${ }^{\circledR}$ Standard 10), used for offline NIR analysis, was supplied by The Dow Chemical Company (Midland, USA).

\subsection{Preparation of the coating suspension}

The coating suspension was prepared with a ratio Aquacoat ECD $30^{\circledR}$ (expressed as dry mass)/Kollicoat $\mathrm{IR}^{\circledR}$ of $90: 10 \mathrm{w} / \mathrm{w}$. The solid
Table 1

Composition of the coating suspension: manufacturing formula for $1 \mathrm{~kg}$ of core tablets.

\begin{tabular}{lclc}
\hline Materials & Suspension $(\mathrm{g})$ & Solid $(\mathrm{g})$ & Percent $(\%)$ \\
\hline${\text { Aquacoat ECD } 30^{\circledR}}^{\circledR}$ & 450.0 & 135.0 & 49.0 \\
Kollicoat IR $^{\circledR}$ & 15.0 & 15.0 & 1.6 \\
Citroflex 2 $^{\circledR}$ & 33.8 & 33.8 & 3.7 \\
Purified water & 420.2 & - & 45.7 \\
Total & 919.0 & 183.8 & 100.0 \\
\hline
\end{tabular}

content of the final suspension was adjusted to $20 \%(\mathrm{w} / \mathrm{v})$ with purified water. The composition of the coating suspension is presented in Table 1. Aquacoat ECD $30^{\circledR}$ was plasticized overnight with Citroflex $2^{\circledR}$. The ratio Citroflex $2^{\circledR} /$ Aquacoat ECD $30^{\circledR}$ (expressed as dry mass) was $1: 4 \mathrm{w} / \mathrm{w}$. Kollicoat IR $^{\circledR}$ was dissolved in purified water before addition to the plasticized Aquacoat ECD $30^{\circledR}$ suspension. The final suspension was mixed for an additional period of $30 \mathrm{~min}$ prior coating and stirred throughout the coating operation using a Rayneri ${ }^{\circledR}$ apparatus (paddle, $200 \mathrm{rpm}$ ). Coating suspension was prepared prior each coating operation.

\subsection{Coating process}

Tablets were coated inside a partially perforated pan coater (Driacoater $500^{\circledR}$, Driam, Eriskirch, Germany). Batch size was $4 \mathrm{~kg}$. Controlled process parameters during coating are listed in Table 2 . The spray nozzle diameter was $0.8 \mathrm{~mm}$. Prior to the coating operation, tablets were preheated at $40{ }^{\circ} \mathrm{C}$ for $20 \mathrm{~min}$. Product temperature was maintained at $36^{\circ} \mathrm{C}$ during coating. The process was stopped after spraying $15 \%$ of polymer blend (expressed as percentage of core tablet mass).

Eleven samples were collected at regular intervals, between $5 \%$ and $15 \%$ of theoretical applied polymer blend. Three independent batches were manufactured. At the end of the first coating operation, a sample of dried coating suspension was removed from the inner surface of the pan coater. This sample was analyzed by offline NIR measurements in order to assign specific absorption bands from coated tablet spectral information. In addition, the film coating density was measured by helium pycnometry technique (Micromeretics AccuPyc 1330, Gosford, New South Wales, Australia), from the same dried coating suspension sample in order to determine the theoretical coating thickness derived from the mass of coating materials.

\subsection{Reference measurements}

\subsubsection{Mass of coating materials}

Coated tablets were weighed using a Mettler Toledo AE 50 analytical balance (Mettler Toledo, Colombus, USA). The experimental amount of coating materials was obtained by subtracting the average weight of core tablets from the average weight of coated tablets.

The number of tablets used to calculate the average weights of core tablets and coated tablets was set at 50 tablets to minimize the effect of individual core tablet weight variation.

Table 2

Coating process parameters.

\begin{tabular}{lc}
\hline Process parameters & Values \\
\hline Atomizing air pressure $($ bar $)$ & 2.4 \\
Difference pressure $(\mathrm{mbar})$ & -0.5 \\
Inlet air temperature $\left({ }^{\circ} \mathrm{C}\right)$ & 66 \\
Pan speed $(\mathrm{rpm})$ & 20 \\
Spray rate $(\mathrm{g} / \mathrm{min})$ & 20 \\
\hline
\end{tabular}




\subsubsection{Coating thickness}

Coating layer thickness of tablets was determined by Terahertz Pulsed Imaging (TPI). A TPI Imaga 2000 (TeraView Ltd., Cambridge, UK) was used in off-line mode, equipped with a robotic arm moving the tablet for complete $360^{\circ} 3 \mathrm{D}$ imaging.

The terahertz spectroscopy operates in the far-infrared region of the electromagnetic spectrum $(0.06-3 \mathrm{THz}$, corresponding to a wavelength range of $2-100 \mathrm{~cm}^{-1}$ ) (Zeitler and Gladden, 2008). Coating thickness $\left(d_{\text {coat }}\right)$ can be non-destructively calculated from the terahertz temporal waveform in the time domain, for each analyzed single point, according to Eq. (1) (Ho et al., 2007).

$2 d_{\text {coat }}=\Delta t c / n$

where $\Delta t$ is the time delay between the terahertz pulse reflection occurring at the coated tablet surface and the reflection occurring at the coating/core interface, $n$ is the refractive index value of the coating material and $c$ is the speed of light.

The refractive index of a dried coating suspension sample was determined using Terahertz Pulsed Spectroscopy in the transmission mode. A mean value of $1.38(n=3)$ was found.

A full terahertz map of coated tablets was recorded, comprising approximately 3200 point measurements. For each $200 \mu \mathrm{m}$ $* 200 \mu \mathrm{m}$ pixel, coating thickness was determined. An average coating thickness per tablet was thus calculated. Three tablets per collected sample were analyzed.

\subsection{Near Infrared Spectroscopy (NIRS)}

\subsubsection{NIR equipment and set-up parameters}

Samples were analyzed using a photodiode array spectrometer (MCS 611 NIR 1.7H spectrometer, Carl Zeiss, Germany), equipped with an OMK 500-H reflectance measuring head (wavelength range of $950-1690 \mathrm{~nm}$ ).

A reference measurement using a diffuse white standard of polytetrafluoroethylene (PTFE, Spectralon ${ }^{\circledR}$ ) was performed prior to each acquisition. The integration time was set at $75 \mathrm{~ms}$. Each spectrum was the average of 20 scans. The total spectrum measurement time was 1500 ms. Acquisition set-up parameters were similar for off-line and in-line acquisitions.

For off-line analysis, successive collected samples were positioned on the top of the OMK measuring head in order to cover the entire measurement surface. For in-line analysis, the integration of the OMK measuring head inside the rotating drum was carried out without interfering with the coating process, as shown in Fig. 1. The probe was positioned at the front of the drum, parallel to the spray to prevent clogging of the reading surface. The optimum clearance between the OMK head and the analyzed area was set at $15 \mathrm{~mm}$. In-line NIR spectra acquisitions were performed every $3 \mathrm{~s}$, without interrupting the coating process, until the required mass of coating materials was sprayed. At each sampling time performed while the theoretical applied polymer blend was 5-15\%, $10 \mathrm{NIR}$ spectra (bracketing the corresponding sampling time) were

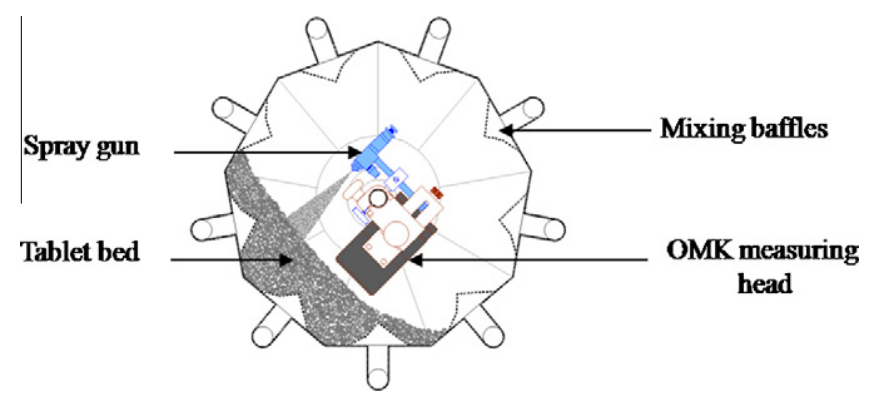

Fig. 1. Schematic integration of the probe inside the pan coater. selected to perform multivariate analysis. This methodology minimized the influence of tablet bed moving (due to drum rotation and mixing baffles) on spectral information and allowed a representative sampling of coated tablets.

ProcessXplorer $^{\circledR}$ software (version 1.2.0.30, Carl Zeiss, Germany) was used to collect NIR spectra. All NIR data were exported from processXplorer ${ }^{\circledR}$ software and analyzed using Matlab ${ }^{\circledR}$ 7.8, R2009a (The MathWorks Inc., Natick, MA, USA) software and PLS Toolbox 5.8.3 (Eigenvector Research Inc., Wenatchee, WA, USA).

\subsubsection{Multivariate data analysis}

Data were pre-processed to reduce impacts of light scattering, path length variations and random noise on spectral information, arising from tablet moving and differences in tablet packing. Several pre-processing methods were tested. A Standard Normal Variate (SNV) correction was applied to spectra to correct intensity differences and baseline shifts (Barnes et al., 1989). Derivatives (first and second derivatives) associated with a Savitzky-Golay smoothing algorithm (filter width of 9 points and a second-order polynomial fitting) were also evaluated in order to improve the resolution of overlapping bands and minimize baseline offsets (Savitzky and Golay, 1964).

A multivariate qualitative analysis was performed from in-line pre-processed NIR spectra using Principal Component Analysis (PCA) (Naes et al., 2002), while a multivariate quantitative analysis was carried out using Partial Least Squares (PLS) regression (Wold et al., 2001).

PLS calibration models were evaluated by cross-validation, using a random subset method. The optimum latent variable number was selected with respect to the lowest root mean square error of calibration (RMSEC) and the lowest root mean square error of cross-validation (RMSECV). Equations of these two errors are given below (Naes et al., 2002):

$$
\begin{aligned}
& \text { RMSEC }=\sqrt{\sum_{i=1}^{N}\left(\hat{y}_{i}-y_{i}\right)^{2} /(N-A-1)} \\
& \text { RMSECV }=\sqrt{\sum_{i=1}^{N}\left(\hat{y}_{C V, i}-y_{i}\right)^{2} / N}
\end{aligned}
$$

where $\hat{y}_{i}, y_{i}$ and $\hat{y}_{C V, i}$ correspond to calculated, measured and crossvalidation-estimated values for either mass of coating materials or coating thickness. $N$ is the total number of collected spectra from the calibration set and $A$ represents the number of latent variables used in the PLS model.

The most appropriate PLS models were selected from their predictive ability based on the root mean square error of prediction (RMSEP), which equation is given by (Naes et al., 2002):

$$
\operatorname{RMSEP}=\sqrt{\sum_{i=1}^{N p}\left(\hat{y}_{i}-y_{i}\right)^{2} / N_{p}}
$$

where $N_{p}$ is the number of predicted spectra from the prediction set.

A high value of the correlation coefficient $R^{2}$ was also considered as an indicator of the quality of the PLS models.

Different PLS models were built considering either TPI (model A) or weighing (model B) as reference method. An additional calibration model was created based on the same calibration and prediction sets as in model B but using theoretical coating thicknesses derived from average mass of coating materials, as new reference values (cf. Section 3.3.3) (model C). The objective was to compare NIR predictions obtained from both categories of primary measurements. For the three models, the calibration set was built considering two batches (220 in-line spectra), while an independent batch (110 in-line spectra) was used as prediction set. 


\section{Results and discussion}

\subsection{Off-line NIR analysis}

In order to evaluate the ability of NIRS to monitor the coating of tablets with the studied functional polymer combination, off-line NIR measurements were first carried out.

Superimposition of off-line NIR spectra was performed to assign NIR absorption bands to coating materials or core tablet components and to highlight an increase or decrease in these specific bands. Several studies reported that absorbance values related to coating materials increased while absorbance values related to core tablet components decreased during coating proceeded (Perez-Ramos et al., 2005; Tabasi et al., 2008). Second derivative pre-processed NIR spectra of core tablets and coated tablets for successive collected samples are shown in Fig. 2. The correlation between mass of coating materials or coating thickness and spectral absorbance was clearly highlighted over the $1150-1210 \mathrm{~nm}$ and the $1360-1440 \mathrm{~nm}$ NIR regions (Fig. $2 \mathrm{~A}$ and B, respectively). The overlaid spectrum of the dried coating suspension revealed that these two absorption bands were linked to a component of the coating materials (Fig. 2). The superimposition of the spectra of the dried coating suspension and ethylcellulose powder showed that the spectral information in the dried coating suspension could mostly be explained by ethylcellulose, the main constituent of the coating suspension (Fig. 3). Absorbances over the 1150-1210 nm region were attributed to the $\mathrm{C}-\mathrm{H}$ second overtone, while absorbances over the $1360-1440 \mathrm{~nm}$ region were attributed to the $\mathrm{C}-\mathrm{H}$ stretching and deformation vibrations and to the $\mathrm{O}-\mathrm{H}$ first overtone (Osborne et al., 1993), respectively, and corresponding to functional groups of ethylcellulose.

The increase in absorbances observed in both selected NIR regions, while the applied amount of polymer and the coating thickness increased, was likely linked to the presence of ethylcellulose. Off-line experiments demonstrated clearly the ability of NIR spectroscopy to monitor the tablet coating operation.

\subsection{In-line NIR qualitative monitoring}

Principal component analysis of in-line NIR spectra acquired during the coating operation was performed to discriminate tablets coated with increasing mass of coating materials. In a previous work dealing with HPMC coating and off-line analysis, Roggo et al. (2005) classified samples retrieved at different coating times.

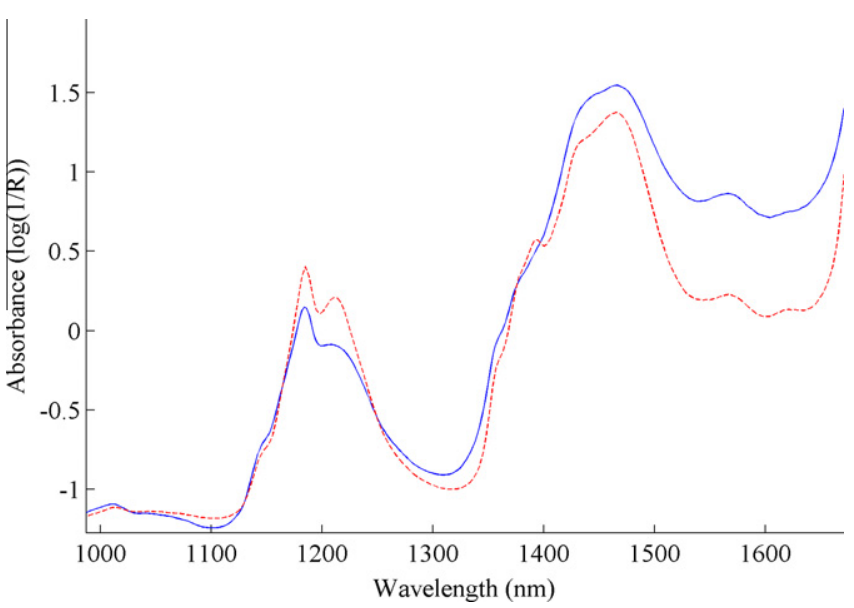

Fig. 3. SNV pre-processed NIR spectra of dried coating suspension (dashed line) and ethylcellulose powder (solid line).

PCA scores on PC2 classified samples in a logical and ordered way related to the theoretical mass of coating materials, as illustrated in Fig. 4. Loadings on PC2 corresponded for a large part to spectral information from the dried coating suspension (Fig. 5). The construction of PC2 could be thus explained by the increase in coating absorbance due to both the coating weight gain and the increase in coating thickness. Implementation of the NIR probe inside the pan coater, associated with the multivariate qualitative spectra analysis, successfully allowed the classification of coated tablets collected at regular intervals during the coating operation.

These results demonstrated the ability of NIRS by in-line measurements to qualitatively monitor the coating operation with the studied functional polymer combination.

\subsection{In-line NIR quantitative monitoring}

3.3.1. Calibration and prediction based on coating thickness

A PLS calibration model was developed from in-line NIR spectra using TPI measurements as primary values (model A). PLS results for the calibration set are reported in Table 3. The best calibration model was obtained using a second derivative transformation of in-line spectra and considering both NIR regions of interest. A 3-latent variable PLS calibration model was generated yielding both
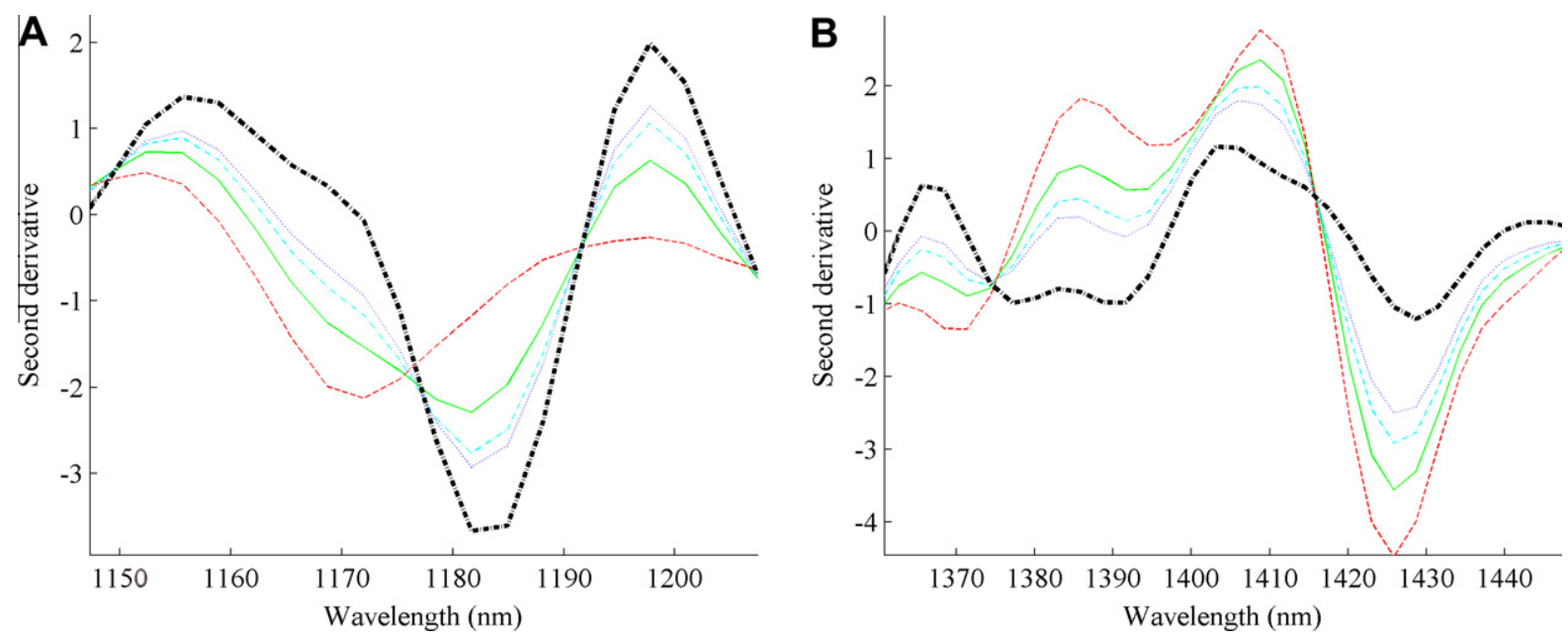

Fig. 2. Second derivative pre-processed NIR spectra of core tablets (dashed line), dried coating suspension (bold dash-dotted line) and coated tablets for various theoretical mass of coating materials: $12 \mathrm{mg}$ (solid line), $25 \mathrm{mg}$ (dash-dotted line), $37 \mathrm{mg}$ (dotted line), (A) in the 1150-1210 $\mathrm{nm}$ and (B) in the $1360-1440 \mathrm{~nm}$ NIR regions. 


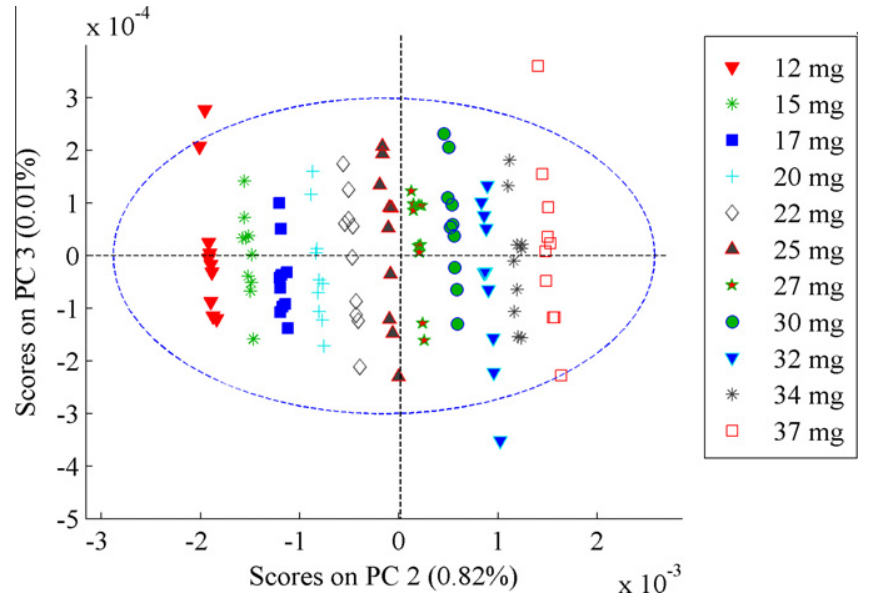

Fig. 4. PCA scores plot obtained from second derivative transformation of in-line NIR spectra, from one batch. The theoretical mass of coating materials for the collected samples ranged from 12 to $37 \mathrm{mg}$.

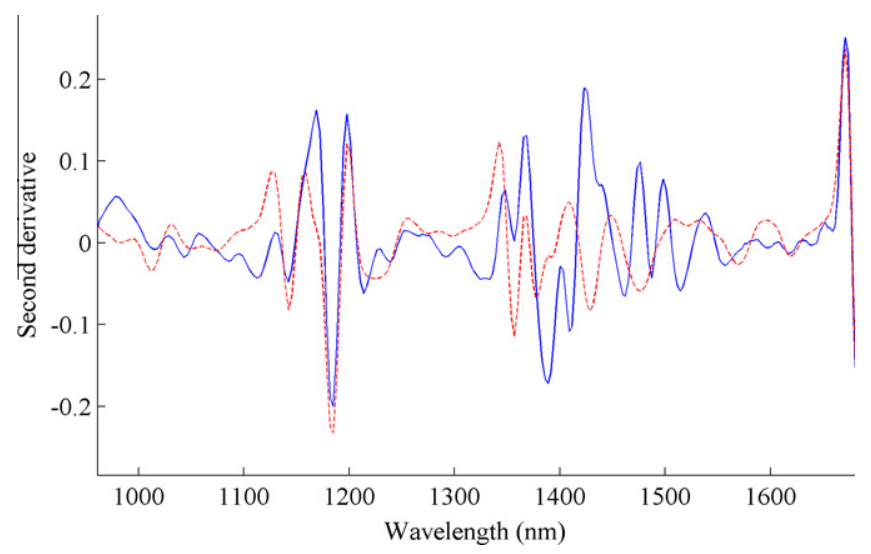

Fig. 5. Superimposition of second derivative pre-processing of dried coating suspension spectral information (dashed line), and PCA loadings plot corresponding to scores on PC2 (solid line)

low calibration error and cross calibration error, associated with a high $R^{2}$. An independent batch was prepared to evaluate the predictive ability of the calibration model. PLS coating thickness predictions gave a RMSEP of $4.02 \mu \mathrm{m}$ (Table 3 ). The low predictive error compared to the actual increase of coating thickness between successive sampling times (around $12 \mu \mathrm{m}$ ) demonstrated the validity of the calibration model. Coating thickness predictions were in agreement with TPI coating thickness values as a linear relationship with a high $R^{2}$ was found in the actual thickness range of 60 and $170 \mu \mathrm{m}$. As recently suggested by Maurer and Leuenberger (2009) who compared TPI and NIR imaging to monitor a tablet coating operation, the present study demonstrated efficient calibration of NIR measurements with TPI coating thickness. Only three tablets per sampling time were required for accurately determination of coating thickness. TPI did not account for the variability in the dimensions of the core tablets thus allowing the determination of more accurate coating thickness values.

This work demonstrated the interest of TPI as innovative and non-destructive reference method, in comparison with other film thickness determination methods such as ordinary micrometric measurements (Perez-Ramos et al., 2005; Tabasi et al., 2008) or more sophisticated image analysis (Andersson et al., 2000; Lee et al., 2011; Možina et al., 2010).

\subsubsection{Calibration and prediction based on mass of coating materials}

Weighing was used as another reference method to create a calibration model from in-line NIR spectra (model B). This model was developed based on the same sampling range and on the same number of in-line NIR spectra as in model A. Even though this primary method is a fast and simple approach requiring no sample preparation, the major drawback in terms of accuracy remains its dependency to core tablet weight uniformity. Therefore, the influence of core tablet weight variability on reference value accuracy should be minimized. For this purpose, more coated tablets per sampling time were analyzed compared to model A (50 tablets instead of 3 tablets). PLS results for calibration set are presented in Fig. 6A. The optimum calibration model was again obtained using a second derivative pre-processing of in-line spectra and considering both NIR regions of interest. A 2-latent variable PLS calibration model was created providing both low calibration error and cross calibration error, associated with a high $R^{2}$. The predictive ability of the calibration model was evaluated using an independent batch. PLS coating weight predictions gave a RMSEP of $0.91 \mathrm{mg}$ (Fig. 6B). The low predictive error compared to the actual increase of mass between successive sampling times (around $2.5 \mathrm{mg}$ ) demonstrated the validity of the calibration model. As previously seen for model $\mathrm{A}$, a linear relationship with a high $R^{2}$ was found for model $\mathrm{B}$, in the actual mass range of 11 and $35 \mathrm{mg}$, demonstrating the correlation between actual and calculated values. The ability of in-line NIR measurements to monitor in real-time the coating operation was depicted in Fig. 7. The real-time NIR predictions calculated at each NIR acquisition were similar to the actual mass of coating materials determined by weighing.

Previous studies described off-line or at-line NIR measurements to monitor the mass of coating materials (Han and Faulker, 1996; Kirsch and Drennen, 1996). The present work demonstrated that in-line NIR measurements combined with the multivariate quantitative analysis allowed successfully to monitor the amount of coating materials applied to tablets.

\subsubsection{Comparison of NIR prediction accuracy according to the applied reference method}

To our knowledge, no study reported a comparison of NIR prediction accuracy obtained using either coating thicknesses or mass of coating materials as primary values.

To carry out this comparison, errors of prediction had to be expressed in the same unit. Coating weights $w_{\text {coating, }}$ used as primary values to calibrate NIR spectral information in model B, were thus mathematically transformed into coating thicknesses $d_{\text {math }}$, as described below. The coating weight is directly related to the volume

Table 3

PLS calibration model and PLS prediction results for model A (TPI as reference method) and model C (weighing followed by mathematical transformation as reference method)

\begin{tabular}{|c|c|c|c|c|c|c|c|c|c|c|}
\hline \multirow[t]{2}{*}{ Models } & \multicolumn{6}{|l|}{ Calibration } & \multicolumn{4}{|c|}{ Prediction } \\
\hline & $\begin{array}{l}\text { Latent variables included in PLS } \\
\text { regression }\end{array}$ & $\begin{array}{l}\text { RMSEC } \\
(\mu \mathrm{m})\end{array}$ & $\begin{array}{l}\text { RMSECV } \\
(\mu \mathrm{m})\end{array}$ & $R^{2}$ & Slope \pm SD & $\begin{array}{l}\text { Intercept } \pm S D \\
(\mu \mathrm{m})\end{array}$ & $\begin{array}{l}\text { RMSEP } \\
(\mu \mathrm{m})\end{array}$ & $R^{2}$ & Slope \pm SD & $\begin{array}{l}\text { Intercept } \pm \mathrm{SD} \\
(\mu \mathrm{m})\end{array}$ \\
\hline A & 3 & 3.82 & 3.88 & 0.988 & $0.99 \pm 0.01$ & $1.38 \pm 0.90$ & 4.02 & 0.988 & $0.97 \pm 0.01$ & $2.56 \pm 1.22$ \\
\hline C & 2 & 2.82 & 2.85 & 0.994 & $0.99 \pm 0.01$ & $0.71 \pm 0.64$ & 4.29 & 0.992 & $0.99 \pm 0.01$ & $-1.54 \pm 1.01$ \\
\hline
\end{tabular}



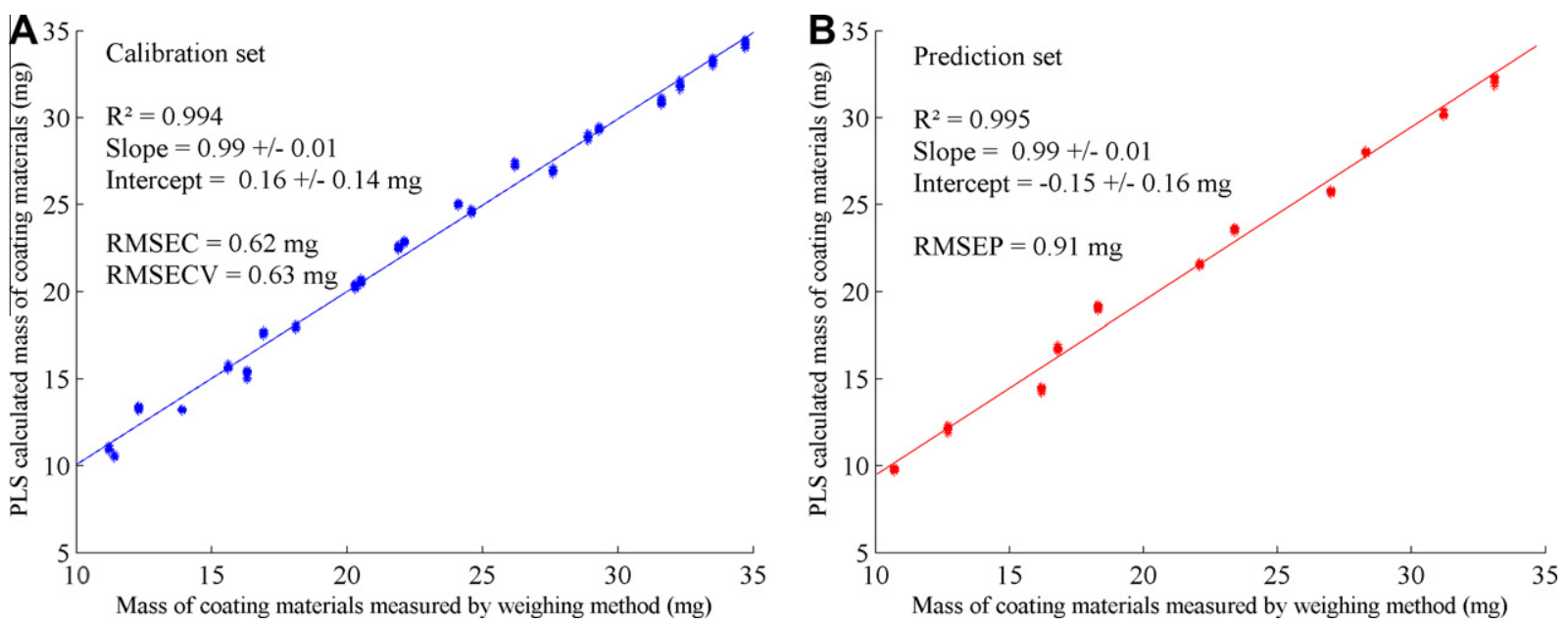

Fig. 6. PLS results based on in-line NIR spectra and mass of coating materials: model B, (A) calibration, (B) prediction.

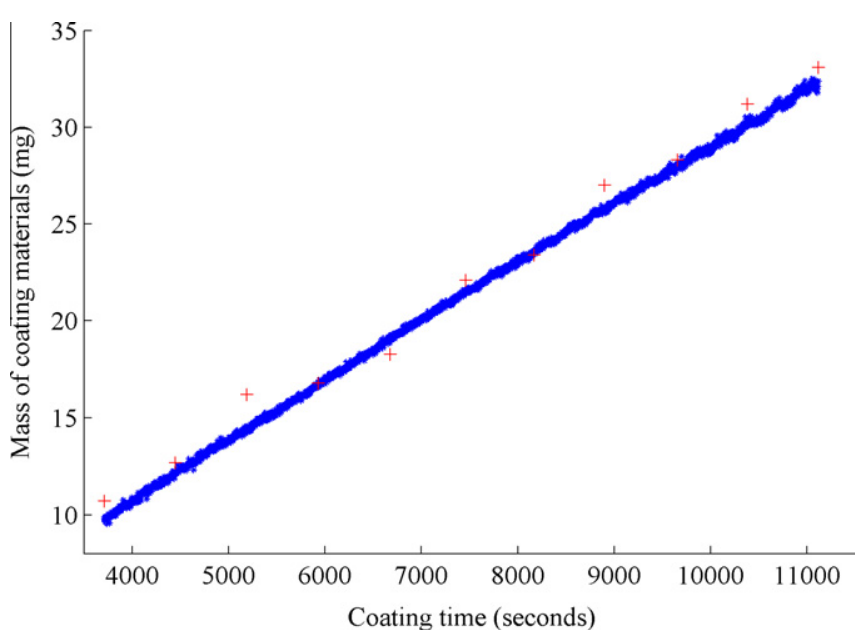

Fig. 7. Real-time monitoring of coating deposit mass: $(*)$ real-time NIR predictions during the pan coating operation and $(+)$ experimental results obtained by weighing.

of the coating applied to the tablet $V_{\text {coating }}$ and to the film coating density $\rho_{\text {coating, }}$ according to the following relation:

$w_{\text {coating }}=\rho_{\text {coating }} \cdot V_{\text {coating }}$

Film coating density was measured from a sample of dried coating suspension removed from the inner surface of the pan coater and a value of $1.17 \mathrm{~g} \mathrm{~cm}^{-3}$ was found $(n=3)$.

The volume of the coating applied to the tablet was calculated by subtracting the volume of the core tablet $V_{\text {core tablet }}$ from the volume of the coated tablet $V_{\text {coated tablet, }}$ according to Eq. (6).

$V_{\text {coating }}=V_{\text {coated tablet }}-V_{\text {core tablet }}$

The volume of a round biconvex tablet is derived from the volumes of a cylinder and two spherical segments of known curvature radius (CR) (Bauer et al., 1998). Assuming coating thickness was uniform around the tablet, the volumes of core and coated tablet were thus given by the following equations:

$V_{\text {core tablet }}=\pi\left(r^{2} s+r^{2} h+3 h^{3}\right)$

$V_{\text {coated tablet }}=\pi\left\{\left(r+d_{\text {math }}\right)^{2} s+\left(r+d_{\text {math }}\right)^{2}\left(h+d_{\text {math }}\right)+3\left(h+d_{\text {math }}\right)^{3}\right\}$

where $r$ is the radius of the cylindrical part, $s$ and $h$ are the height of the cylindrical part and the height of the spherical cap, respectively.
$V_{\text {coating }}$ was obtained by replacing Eqs. (7) and (8) into Eq. (6), leading to the following equation:

$$
\begin{aligned}
V_{\text {coating }}= & \pi\left\{4 d_{\text {math }}^{3}+(s+2 r+10 h) d_{\text {math }}^{2}+\left(2 r s+2 h r+r^{2}\right.\right. \\
& \left.\left.+9 h^{2}\right) d_{\text {math }}\right\}
\end{aligned}
$$

Theoretical coating thickness values $d_{\text {math }}$ were finally calculated by replacing Eq. (9) into Eq. (5) and by solving the resulting cubic equation:

$$
\begin{aligned}
& 4 d_{\text {math }}^{3}+(s+2 r+10 h) d_{\text {math }}^{2}+\left(2 r s+2 h r+r^{2}+9 h^{2}\right) d_{\text {math }} \\
& \quad=w_{\text {coating }} /\left(\rho_{\text {coating }} \pi\right)
\end{aligned}
$$

Using theoretical coating thickness values, an additional calibration model was built (model C). Calibration and prediction sets as well as pre-processing of in-line NIR spectra and selected NIR regions remained identical to the initial model. PLS calibration model and PLS prediction results are summarized in Table 3. Results obtained from both reference methods appeared similar. Both TPI and weighing could successfully be used as reference method in order to calibrate in-line NIR spectral information, providing accurate NIR predictions of coating thickness and deposit mass.

\section{Conclusions}

The aim of this study was to carry out a real-time pan coating monitoring by near infrared spectroscopy. Film thickness and mass of coating materials were selected as monitored critical attributes. The functional polymer blend presently used has never been described for tablet coating. Off-line spectra analysis revealed the ability of NIRS to monitor the tablet coating by focussing on the increase in coating absorbance related to ethylcellulose, the main constituent of the coating suspension. Real-time monitoring of the coating operation was then ensured by implementation of a NIR probe inside a rotating drum coater ( $4 \mathrm{~kg}$ batch size), without interrupting the process. A multivariate qualitative analysis of inline NIR spectra classified in a logical order the coated tablets collected at regular intervals throughout the coating operation. Calibration of NIR spectral information was successfully carried out using either TPI or weighing as reference method. This demonstrated the interest of TPI as innovative and non-destructive reference method, since it does not account for the variability in core tablet dimensions and required only three tablets to get reliable results. Alternatively, weighing can also be used as simple and fast reference method provided at least 50 tablets are weighed. 
A comparison between PLS results demonstrated that both primary methods led to models of similar quality.

In-line NIR measurements associated with multivariate analyses fulfilled the expectations of ICH Q8 guideline on pharmaceutical development, especially in terms of PAT control strategy and reduced end-product (coated tablet) testing. Production scale adaptation (100 kg batch size) is now in progress.

\section{Acknowledgements}

The authors acknowledge TeraView Ltd. for Terahertz Pulsed Imaging measurements.

Claire Gendre is supported by a CIFRE fellowship granted by Technologie Servier and the French Ministry of Research and Innovation.

\section{References}

Andersson, M., Josefson, M., Langkilde, F.W., Wahlund, K., 1999. Monitoring of a film coating process for tablets using near infrared reflectance spectrometry. J Pharm. Biomed. Anal. 20, 27-37.

Andersson, M., Folestad, S., Gottfries, J., Johansson, M.O., Josefson, M., Wahlund, K., 2000. Quantitative analysis of film coating in a fluidized bed process by in-line NIR Spectrometry and multivariate batch calibration. Anal. Chem. 72, 2099-2108.

Barnes, R.J., Dhanoa, M.S., Lister, S.J., 1989. Standard normal variate transformation and detrending of near infrared diffuse reflectance. Appl. Spectrosc. 43 (5), 772 779.

Bauer K.H. Lehmann, K. Osterwald, H.P. Rothgang G, 1998 Coated Pharmaceutical Dosage Form: Fundamentals, Manufacturing Techniques, Biopharmaceutical Aspects, Test Methods and Raw Materials. Medpharm $\mathrm{GmbH}$, Scientific Publishers, Stuttgart.

Cahyadi, C., Karande, A., Chan, L., Heng, P., 2010. Comparative study of nondestructive methods to quantify thickness of tablet coatings. Int. J. Pharm. 398 39-49.

Cogdill, R.P., Forcht, R., Shen, Y., Taday, P.F., Creekmore, J., Anderson, C.A., Drennen, J.K., 2007. Comparison of terahertz pulse imaging and near-infrared spectroscopy for rapid, non-destructive analysis of tablet coating thickness and uniformity. J. Pharm. Innov. 2 (1), 29-36.

Fitzgerald, A.J., Cole, B.E., Taday, P.F., 2005. Nondestructive analysis of tablet coating thickness using Terahertz Pulsed Imaging. J. Pharm. Sci. 94 (1), 177-183.

Food and Drug Administration, 2004. Guidance for Industry. PAT - A Framework for Innovative Pharmaceutical, Development, Manufacturing and Quality Assurance.

Han, S.M., Faulker, P.G., 1996. Determination of SB 216469-S during table production using near-infrared reflectance spectroscopy. J. Pharm. Biomed. Anal. 14 (12), 1681-1689.

Ho, L., Müller, R., Römer, M., Gordon, K., Heinämäki, J., Kleinebudde, P., Pepper, M. Rades, T., Shen, Y., Strachan, C., Taday, P., Zeitler, J., 2007. Analysis of sustainedrelease tablet film coats using terahertz pulsed imaging. J. Control. Release 119, 253-261.

International Conference on Harmonisation of Technical Requirements for Registration of Pharmaceuticals for Human Use, 2009. Pharmaceutical Development Q8 (R2)

Kirsch, J.D., Drennen, J.K., 1995. Determination of film-coated tablet parameters by near-infrared spectroscopy. J. Pharm. Biomed. Anal. 13, 1273-1281.

Kirsch, J.D., Drennen, J.K., 1996. Near-Infrared Spectroscopic monitoring of the film coating process. Pharm. Res. 13, 234-237.
Larsen, C.C., Sonnergaard, J.M., Bertelsen, P., Holm, P., 2003. Validation of an image analysis method for estimating coating thickness on pellets. Eur. J. Pharm. Sci. 18, 191-196.

Lee, M.-J., Park, C.-R., Kim, A.-Y., Kwon, B.-S., Bang, K.-H., Cho, Y.-S., Jeong, M.-Y., Choi, G.-J., 2010. Dynamic calibration for the in-line NIR monitoring of thickness of pharmaceutical tablets processed in a fluid-bed coater. J. Pharm. Sci. 99, 325335.

Lee, M.-J., Seo, D.-Y., Lee, H.-C., Wang, I.-C., Kim, W.-S., Jeong, M.-Y., Choi, G.-J., 2011 In-line NIR quantification of film thickness on pharmaceutical pellets during a fluid bed coating process. Int. J. Pharm. 403, 66-72.

Maurer, L., Leuenberger, H., 2009. Terahertz pulsed imaging and near infrared imaging to monitor the coating process of pharmaceutical tablets. Int. J. Pharm. $370,8-16$.

McGinity, J.W., Felton, L.A., 2008. Aqueous Polymeric Coatings for Pharmaceutical Dosage Forms, third ed. Informa Healthcare, New York.

Moes, J.J., Ruijken, M.M., Gout, E., Frijlink, H.W., Ugwoke, M.I., 2008. Application of process analytical technology in tablet process development using NIR Spectroscopy: blend uniformity, content uniformity and coating thickness measurements. Int. J. Pharm. 357, 108-118.

Možina, M., Tomaževič, D., Leben, S., Pernuš, F., Likar, B., 2010. Digital imaging as a process analytical technology tool for fluid-bed pellet coating process. Eur. J. Pharm. Sci. 41 (1), 156-162.

Muschert, S., Siepmann, F., Cuppok, Y., Leclercq, B., Carlin, B., Siepmann, J., 2009. Improved long term stability of aqueous ethylcellulose film coatings: importance of the type of drug and starter core. Int. J. Pharm. 368, 138-145.

Naes, T., Isaksson, T., Fearn, T., Davies, T., 2002. A User-Friendly Guide to Multivariate Calibration and Classification. NIR Publications, Chichester.

Osborne, B.G., Fearn, T., Hindle, P.H., 1993. Practical NIR spectroscopy with applications in food and beverage analysis, second ed. Longman Group.

Perez-Ramos, J., Findlay, W., Peck, G., Morris, K., 2005. Quantitative analysis of film coating in a pan coater based on in-line sensor measurement. AAPS PharmSciTech 6, E127-E136.

Porter, S., Sackett, G., Liu, L., 2009. Development, optimization and scale-up of process parameters: pan coating. In: Qiu, Y., Chen, Y., Zhang, G.G.Z. (Eds.), Developing Solid Oral Dosage Forms: Pharmaceutical Theory and Practice, first ed. Academic Press, New York, pp. 761-805.

Reich, G., 2005. Near Infrared Spectroscopy and imaging: basic principles and pharmaceutical application. Adv. Drug Deliv. Rev. 57, 1109-1143.

Roggo, Y., Jent, N., Edmond, A., Chalus, P., Ulmschneider, M., 2005. Characterizing process effects on pharmaceutical solid forms using near-infrared spectroscopy and infrared imaging. Eur. J. Pharm. Biopharm. 61, 100-110.

Roggo, Y., Chalus, P., Maurer, L., Lema-Martinez, C., Edmond, A., Jent, N., 2007. A review of near infrared spectroscopy and chemometrics in pharmaceutical technologies. J. Pharm. Biomed. Anal. 44 (3), 683-700.

Römer, M., Heinämäki, J., Strachan, C., Sandler, N., Yliruusi, J., 2008. Prediction of tablet film-coating thickness using a rotating plate coating system and NIR spectroscopy. AAPS PharmSciTech 9 (4), 1047-1053.

Savitzky, A., Golay, M.J., 1964. Smoothing and differentiation of data by simplified least squares procedures. Anal. Chem. 36, 1627-1639.

Siepmann, F., Hoffmann, A., Leclercq, B., Carlin, B., Siepmann, J., 2007. How to adjust desired drug release patterns from ethylcellulose-coated dosage forms. J. Control. Release 119, 182-189.

Tabasi, S.H., Fahmy, R., Bensley, D., O’Brien, C., Hoag, S.W., 2008. Quality by design: Part II. Application of NIR spectroscopy to monitor the coating process for a pharmaceutical sustained release product. J. Pharm. Sci. 97 (9), 4052-4066.

Wold, S., Sjöström, M., Eriksson, L., 2001. PLS-regression: a basic tool of chemometrics. Chem. Int. Lab. Syst. 58, 109-130.

Zeitler, J.A., Gladden, L.F., 2008. In-vitro tomography and non-destructive imaging at depth of pharmaceutical solid dosage forms. Eur. J. Pharm. Biopharm. 71, 2-22.

Zeitler, J.A., Shen, Y., Baker, C., Taday, P.F., Pepper, M., Rades, T., 2007. Analysis of coating structures and interfaces in solid oral dosage forms by three dimensional terahertz pulsed imaging. J. Pharm. Sci. 96 (2), 330-340. 